\title{
Thermal degradation of biological DNA studied by dielectric spectroscopy
}

\author{
Jacek Nizioł $^{\mathrm{a}, *}$, Robert Ekiert ${ }^{\mathrm{b}}$, Justyna Kuczkowska ${ }^{\mathrm{c}}$, Patryk Fryń $^{\mathrm{c}}$, Monika Marzec ${ }^{\mathrm{c}}$ \\ ${ }^{\text {a }}$ AGH University of Science and Technology, Faculty of Physics and Applied Computer Science, Mickiewicza 30, 30-059, Kraków, Poland \\ ${ }^{\mathrm{b}}$ Department of Molecular Biophysics, Faculty of Biochemistry, Biophysics and Biotechnology, Jagiellonian University, Gronostajowa 7, 30-387, Kraków, Poland \\ ${ }^{\mathrm{c}}$ Institute of Physics, Jagiellonian University, Lojasiewicza 11, 30-348, Kraków, Poland
}

\section{A R T I C L E I N F O}

\section{Keywords:}

DNA

Deoxyribonucleic acid

Melting

Dielectric spectroscopy

Thermal stability

\begin{abstract}
A B S T R A C T
Dielectric spectroscopy was tested as an alternative tool to study degradation of deoxyribonucleic acid (DNA) in its solid form. The specimens, prepared from biological DNA, were periodically heated and cooled according to a programmed scheme. Simultaneously, their dielectric parameters (permittivity and dielectric loss) were monitored as function of frequency and temperature. The analysis of Bode plots allowed to determine the upper limit of thermal stability of solid DNA at $120^{\circ} \mathrm{C}$, because heating at higher temperatures resulted in irreversible changes. These changes were identified as denaturation by gel electrophoresis and UV-vis absorption methods.
\end{abstract}

\section{Introduction}

The deoxyribonucleic acid (DNA) is a macromolecule of fundamental importance for all living organisms. Many unique chemical and physical properties distinguish DNA from synthetic polymers, due to its ability to maintain the structure of the double-helix.

This fact, since the beginning of the last decade, has motivated the search for new applications in fields outside of the life sciences. In such applications, DNA is used as a bulk material, in its natural form or in a complex with an amphiphilic (surfactant) molecule. These materials in the form of thin layers have been added to the structure of devices classified as organic electronics, optoelectronics and non-linear optics. As a consequence, a significant improvement in performance was observed. An extensive discussion of non-standard DNA applications can be found in recent review articles, for example in [1,2].

DNA is a highly hydrophilic biopolymer [3,4], what directly impacts on its morphology [5,6]. In the case of DNA complexes, aliphatic (and so hydrophobic) tails of surfactant molecules extend to outermost directions, making possible to dissolve the complex in some organic solvents [7]. However, even this hydrophobic cocoon does not protect entirely the DNA backbone from water molecules penetrating from the surrounding atmosphere [8-10]. Water molecules confined in either DNA or DNA complex belong to a one of two pools, according to the strength and the character of the binding mechanism. The first shell, referred to as tightly bound water, consists of molecules directly bound via hydrogen bonds to hydrophilic sites on the DNA helix. To the other one, known as loosely bound water, belong water molecules attached to tightly bound waters [11]. These waters can be the source of uncontrolled ionic conduction that may occur extremely detrimental for performance of organic electronics devices. A range of techniques is used to dry DNA, like for example freeze-drying or prolonged equilibration in dry atmosphere. However, even prolonged drying under vacuum in presence of $\mathrm{P}_{2} \mathrm{O}_{5}$ is insufficient to entirely dry DNA and leaves a few weight percent of water [12]. Thin films of DNA for organic electronics are processed from solution. Such films, even if initially dried, have to be transferred to an evaporating system, where other layers are deposited. During the transfer, the DNA layer risks an extra contamination with atmospheric humidity. For this reason, the film is heated inside the evaporator to remove residual water or solvent. The question arises, what is the actual temperature, that DNA is able to withstand without irreversible changes concerning its principal morphology. The double helix of DNA, where planar base pairs are arranged in equidistant stacks, is believed to determine electrical charge transport [13]. The double helix structure is also vital for effective hosting dye molecules used in DNA-based nonlinear optics [14,15].

The structure of the double helix under the influence of factors such as $\mathrm{pH}$ or temperature, undergoes denaturation process [16], also called DNA melting. Its source resides in the disruption of Watson-Crick base-pairs. During the DNA melting occur local openings and reclosing of the double-stranded helix, referred to as breathing fluctuations [16]. In the course of such a process, the DNA molecule is sorely deformed. The double helix is locally stretched, bended, untwisted or compressed [17]. The structural change is crisp, and virtually no stable intermediate state exists between the fully native and fully denatured states. This

\footnotetext{
* Corresponding author.

E-mail address: niziol@agh.edu.pl (J. Nizioł).
} 
process can be classified as one-dimensional first-order phase transition [18]. At the end bases lose their stacking and the specific helical form of the backbone disappears [19]. The reverse process known as renaturation is also possible for oligomeric DNA under special, carefully controlled conditions. In a buffer solution, DNA dissociates in two separate strands between $70{ }^{\circ} \mathrm{C}$ and $90^{\circ} \mathrm{C}$, depending on ratio of A-T and G-C base-pairs, helix length etc. [16]. However, in the solid form of DNA, due to lack of excess water, this phenomenon is supposed to occur at higher temperatures.

In polymer science a standard tool used to detect thermal decomposition is thermogravimetric analysis (TGA). Many researchers locate DNA degradation at the temperature equal c.a. to $220^{\circ} \mathrm{C}$, where occurs an abrupt loss of the sample mass [20,21]. As can be seen in Fig. 1, in a thermogram of DNA four regions can be discerned by the derivative of the mass loss.

The first one, below $100^{\circ} \mathrm{C}$, the second between $100^{\circ} \mathrm{C}$ and $150{ }^{\circ} \mathrm{C}$, the third between $150^{\circ} \mathrm{C}$ and $220^{\circ} \mathrm{C}$ and the last one is located above $220^{\circ} \mathrm{C}$. These ranges may vary, depending on experimental conditions. At temperatures inferior to $220^{\circ} \mathrm{C}$, the gas released by the heated DNA, is only water vapour [22]. Intuitively it can be assumed, that loosely bound water is removed in the first region, the tightly bound water in the second one, while nothing particular happens in the third region.

However, as it was demonstrated in [23], elevated temperatures induce shortening of long chain DNA (2686 base pairs - bp) starting already from $130^{\circ} \mathrm{C}$. On the other hand, the authors detected content of the double helix DNA even in the specimen heated at $200^{\circ} \mathrm{C}$. Nevertheless, the role of short heating time (single minutes) and the used protocol should be emphasised here.

In the case of DNA dissolved in water, the melting temperature can be easily determined according to well established protocols [16]. On the contrary, melting occurring in other forms of DNA still remains the subject of research and debate. Different alternative approaches has already been proposed. For example melting of double strand DNA macromolecules anchored to surfaces were studied by surface plasmon resonance technique (FO-SPR) [24] or electrochemical methods [25]. Denaturation of separated DNA helices deposited on a support was observed by atomic force microscopy [26], while that occurring in bulk samples of solid DNA neutron scattering [27], differential scanning calorimetry (DSC) [28] or X-Ray diffraction [12]. The knowledge about DNA thermal degradation is of wider importance, not only for organic electronics and biotechnology but also for scientifically remote areas like archaeology or forensic science [29,30].

In a previous work [31], we reported on spectroscopic ellipsometry as a convenient tool to analyse thermal degradation of DNA transformed in submicrometer, thin films.

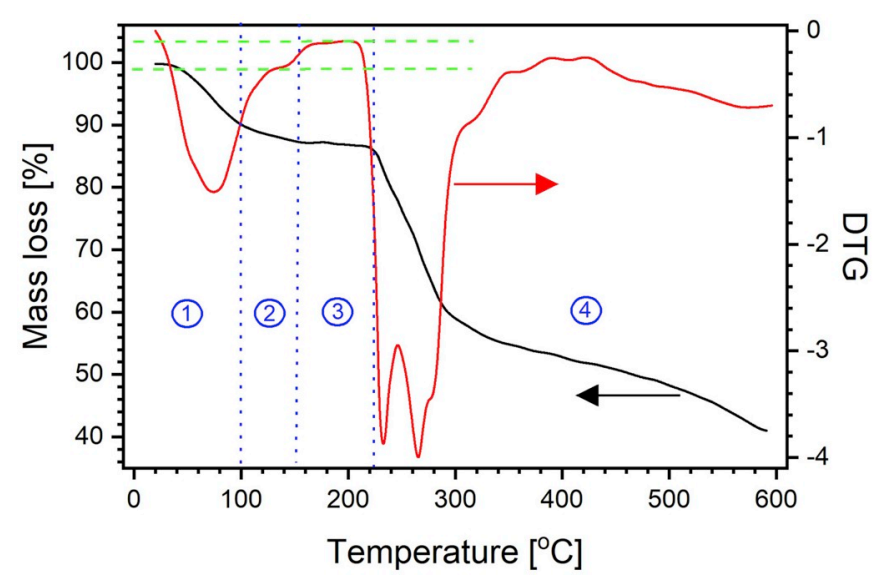

Fig. 1. An example of TGA thermogram of DNA powder. The mass loss curve refers to the left axis, while its derivative, DTG to the right axis (adapted from [22]).
In the current study we continue this research with help of dielectric spectroscopy. Each material, on molecular level, contains electric dipoles of different nature. In external electric field, due to interactions with the molecular surroundings, they behave like damped oscillators. The relaxation time is a measure of the time, such an oscillator needs to regain its equilibrium after it has been disturbed. The distribution of relaxation times allows concluding about occurring molecular phenomena. This constitutes the basis of dielectric spectroscopy, that measures permittivity (denoted as $\varepsilon^{\prime}$ ) and dielectric loss (denoted as $\varepsilon^{\prime \prime}$ ) as function of the field frequency and an environmental factor, most often the temperature [32].

Dielectric spectroscopy has already been applied for DNA testing since pre-Watson-Crick era. However, because of the significance of DNA in bioscience, experiments were mainly limited to aqueous solutions. Nevertheless, the knowledge about relaxation processes occurring in DNA is still incomplete.

An exhaustive review of experimental findings and postulated theoretical models can be found in [33]. The main conclusions presented there can be summarized as follows. DNA double helix does not have a permanent net dipole moment. In the literature, the model qualitatively describing the dielectric DNA response is referred to as Manning-Mandel-Oosawa model. It assumes that once DNA is dissolved in an aqueous solution, some of the negative charge of the molecule is neutralized by cations, especially in saline solutions. In effect a counterion layer forms around the DNA molecules. The counterions are non-localized, what implies that the positive ions can move relatively freely along the DNA surface and within the surrounding hydration shell. Therefore, the dipole moment of DNA occurs as a result of distortion of the counterion layer, forced by external electrical voltage. Typically, in aqueous solution of DNA, three relaxation processes are observed - at several $\mathrm{kHz}$, between $1 \mathrm{MHz}$ and $1 \mathrm{GHz}$ and the last one above $1 \mathrm{GHz}$. The exact values vary, depending on the material and experimental conditions [33].

However, it is more common to use dielectric spectroscopy limited to low and medium radio frequencies (below a few $\mathrm{MHz}$ ) as the tool for solid polymers characterization [32].

To interpret results of dielectric spectroscopy it is customary to draw so-called Bode plots that show $\varepsilon^{\prime}$ and $\varepsilon$ " against frequency in double logarithmic scale. Several typical features of the curves allow a rough classification of the phenomena that are behind them. In vicinity of the frequency corresponding to a relaxation process, $\varepsilon^{\prime}$ decreases in a steplike manner. A strong increase of $\varepsilon^{\prime}$ at low frequencies is due to electrode polarization. On the other hand, $\varepsilon$ " exhibits a maximum at the relaxation frequency. Its linear increase with decreasing frequency is explained by ohmic-like conductivity. Maxima appearing in $\varepsilon$ " Bode plot from high to low frequencies by convention labelled by successive letters of the Greek alphabet, starting from the letter $\alpha$ [32].

In the case of linear polymers at moderate temperatures, most often only $\alpha$-relaxation is observed within this frequency band. This relaxation is associated with micro-Brownian motion of the whole chains [32]. The double strand DNA is a stiff macromolecule of persistence length estimated at c.a. $50 \mathrm{~nm}$ [34]. Thus, the expected movements of its segments in the solid would be seriously hampered. Consequently, if a feature similar to $\alpha$-relaxation occurs in the dielectric spectrum of heated DNA, it can be interpreted as the presence of single chains that have emerged as a result of the decomposition of the double helix.

\section{Materials}

Low molecular weight DNA (purity 98\%) obtained from salmon sperm and other chemicals were provided by Sigma-Aldrich and used without further purification. Stock of Tris-HCl (Tris - tris(hydroxylmethyl)aminomethane) buffer was prepared at $1 \mathrm{M}$ concentration with $\mathrm{pH}$ adjusted to 8. DNA was dissolved in the buffer to obtain $5 \mathrm{mg} /$ $\mathrm{ml}$ DNA in $10 \mathrm{mM}$ Tris-HCl. For electrophoresis TBE buffer $(0.089 \mathrm{M}$ Tris, 0.089 M Boric acid, 0.002 M EDTA) was used. 
DNA fibers were freeze-dried at $-53^{\circ} \mathrm{C}$, under $0.056 \mathrm{mbar}$ for $18 \mathrm{~h}$ and stored in tightly closed container. Next, it was compressed in three tablets ( $6 \mathrm{~mm}$ diameter, thickness c.a. $0.5 \mathrm{~mm}$ ), each under different loads - 50, 30 and 20 Bar. During this process and transfer to experimental setup, tablets remained in contact with laboratory atmosphere of normal humidity.

\section{Methods}

\subsection{Electrophoresis}

Electrophoresis was carried out in $3.5 \%(\mathrm{w} / \mathrm{v})$ agarose gel in TBE buffer, following the standard procedure [35] using Mupid-exU apparatus. Reference standards - 50bp and 100bp DNA Step Ladder (Promega) were simultaneously introduced to the gel. The DNA were stained in the gel with a fluorescent dye - ethidium bromide and visualized by illumination with UV light. By this method, molecular mass of the reference and the thermally treated DNA specimens were estimated.

\subsection{UV-vis absorption spectroscopy}

The absorbance of DNA samples in solution was measured on Specord 250 plus spectrophotometer (Analytik Jena) in a $10 \mathrm{~mm}$ quartz cuvette.

\subsection{Dielectric spectroscopy}

Dielectric measurements in the frequency range from $1 \mathrm{~Hz}$ to $2.5 \mathrm{MHz}$ and testing voltage of $0.3 \mathrm{~V}$ were performed using a turn-key Broadband Dielectric Spectrometer (CONCEPT 81, Novocontrol Technologies $\mathrm{GmbH}$ ) equipped with an automatically controlled cryostat (Novocool Cryosystem V.2.2). Samples were measured in plate capacitor geometry, mounted in proprietary holder BDS 1200. The temperature was varied in $10^{\circ} \mathrm{C}$ steps. Rise or decrease of the temperature was carried out at $10^{\circ} \mathrm{C} / \mathrm{min}$ speed, then the temperature was kept constant for next $8 \mathrm{~min}$. This time was empirically established as sufficiently long for the sample to become thermally equilibrated. The heating program was divided in several heating/cooling cycles as it is illustrated in Fig. 2. In the further text the cycles will be named by descriptions used in the diagram.

\section{Results and discussion}

\subsection{Distribution of DNA molecular mass}

DNA extracted from natural cells is a statistic polymer. Bulk quantity

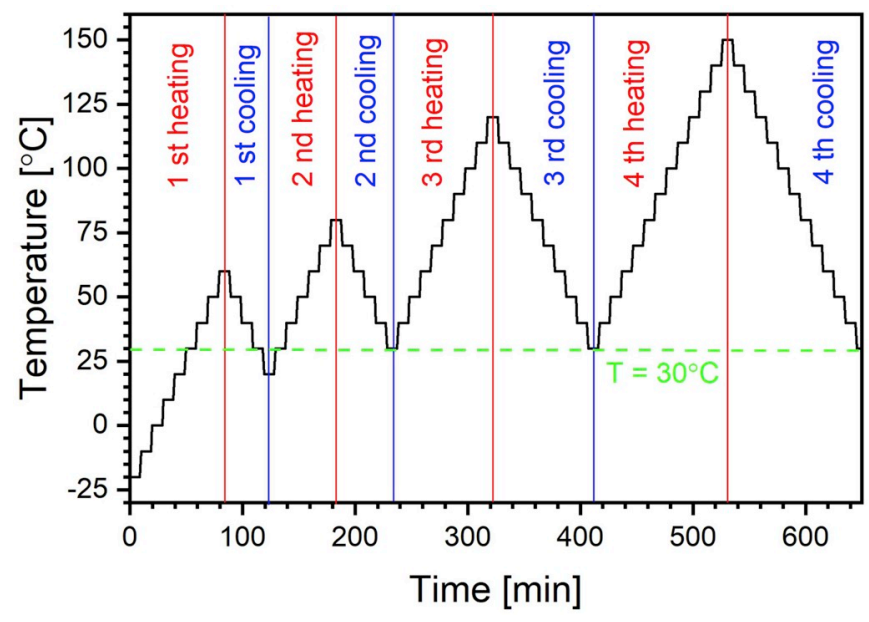

Fig. 2. Scheme of the heating program. consists of shorter and longer macromolecules. Thus, such a DNA is characterized by distributed molecular mass. A range of standard experimental methods is used to determine dispersion of molecular mass in polymers. However, in the case of DNA the most accurate is electrophoresis. A specimen of the tested DNA solution, is introduced to agarose gel. Next, the applied electric field forces the DNA to move. Because shorter macromolecules move faster, the distance travelled in the gel depends on molecular mass.

To calibrate this dependence, a reference sample (so called calibration ladder) of known, discrete molecular mass distribution, travels simultaneously with the studied one. At the end of the protocol, both the ladder and the studied sample are stained with a fluorescent dye and visualized in UV light. A monodispersed molecular mass can be easily identified by reference to the ladder. In the case of a polydispersed molecular mass such a task is more complicated. It can be assumed that staining efficiency is proportional to the number of DNA base pairs that the macromolecule consists of. Hence, the intensity of fluorescence is proportional to the amount of DNA accumulated in the particular region of the gel. Consequently, the polydispersed DNA distributed along the gel can be seen as a streak of gradually varying fluorescence intensity (see the photo in Fig. 3a). The relative intensity at a place chosen in the streak, can be correlated to the located there percent of the total base pairs number of the specimen. Two different ladders in Fig. 3a are in perfect agreement, so positions of their steps can be used to rescale in base pairs units, the distance travelled by the tested DNA. In Fig. 1b are shown fluorescence intensities of $50 \mathrm{bp}$ ladder and the tested DNA as a function of the covered distance, represented in logarithmic scale. Equally spaced peaks from the $50 \mathrm{bp}$ calibration ladder indicate a simple exponential dependence between the distance and the molecular mass. This feature was used to rescale the plot of the tested DNA in terms of relative intensity and base pair number. After simple calculations, there were found for the tested DNA the number average molar mass, $M_{n}=$ $57 \mathrm{bp}=3,75 \cdot 10^{4} \mathrm{~g} \mathrm{~mol}^{-1}$, the mass average molar mass $M_{w}=74 \mathrm{bp}=$ $4.91 \cdot 10^{4} \mathrm{~g} \mathrm{~mol}^{-1}$ and corresponding polydispersity 1.31 .

As the first one, the DNA tablet compacted under 50 bar was subjected to the experimental routine. In Figs. 4-11 are shown Bode plots of both $\varepsilon^{\prime}$ and $\varepsilon^{\prime \prime}$ recorded during heating and cooling cycles from the 1 st to the 4th one. These data will be discussed qualitatively, because of the experiment details. It was not possible to monitor changes of neither its dimensions nor of the quality of the surface in contact with the electrodes. This parameters may have varied, what probably happened in result of the first heating/cooling cycle. Comparison of curves for $\mathrm{T}=30^{\circ} \mathrm{C}$ in Fig. $4 \mathrm{a}$ and $\mathrm{b}$ evidences less intense $\varepsilon$ ' at lower frequencies, while the value at the opposite end remains similar. Such an effect can be explained by smaller electrode polarization due to a better surfaces accommodation between the sample and the electrode caused by heating.

Similarly, in the course of the measurements, water vapour may have permanently left the sample. Indeed, in traces for $\varepsilon$ " shown in Fig. 5a gradually appears a maximum at low frequencies then disappears upon prolonged heating (see Fig. 5b). Such an effect may be associated with the release of excess water. However, a deeper discussion of water dynamics in DNA is beyond the scope of this research. It is worth to underline, that if measured at $30^{\circ} \mathrm{C}$, the shape of the curves that $\varepsilon^{\prime}$ and $\varepsilon^{\prime \prime}$ adopted after the first heating/cooling cycle remains essentially stable after consecutive heating/cooling to $80^{\circ} \mathrm{C}$ (Figs. 6 and 7) and next to $120^{\circ} \mathrm{C}$ (Figs. 8 and 9). As it is shown in Fig. 12 the only difference is a small decrease in values at low frequencies. The relaxation at several $\mathrm{kHz}$ known from the literature [33] does not appear.

A closer inspection of Fig. 9a, illustrating variations of $\varepsilon$ " on heating up to $120^{\circ} \mathrm{C}$, reveals that the general pattern observed up to $80^{\circ} \mathrm{C}$ imitate that shown in Fig. 7a. At temperature of $110^{\circ} \mathrm{C}$ and above emerges a shoulder located at c.a. $10^{5} \mathrm{~Hz}$. The cooling process moves this shoulder leftwards (Fig. 7b) and finally, at $30^{\circ} \mathrm{C}$, the shape of $\varepsilon$ " plot returns to its initial form. The appearance of this shoulder is not 


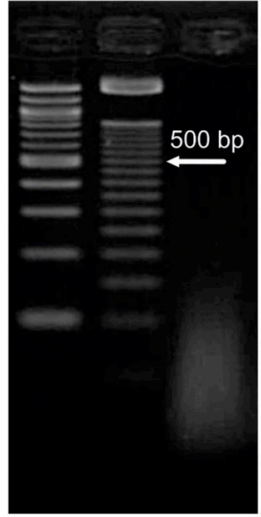

(a)

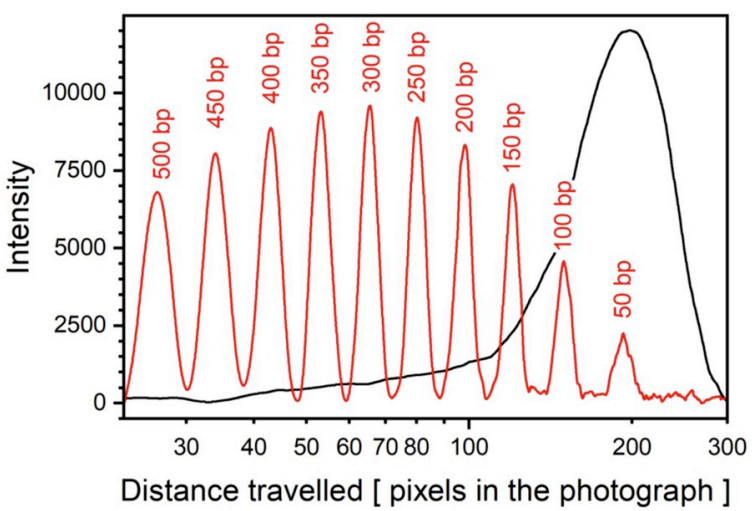

(b)

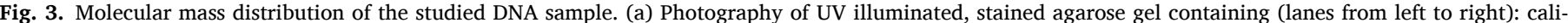

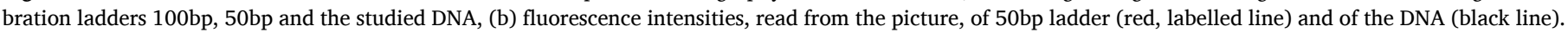

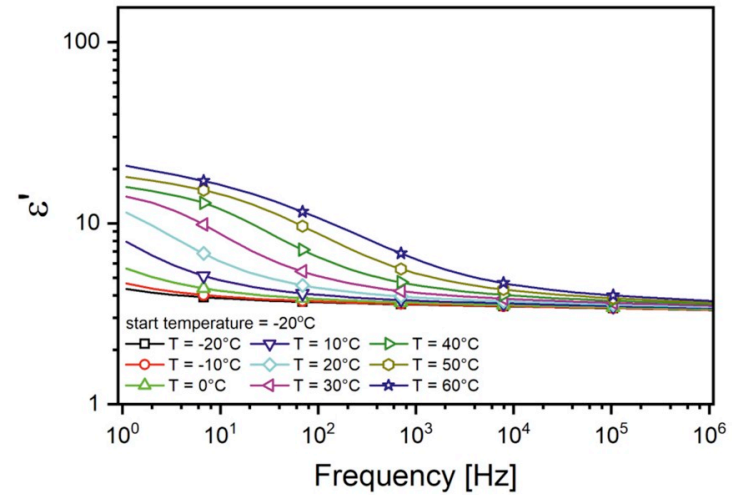

(a)

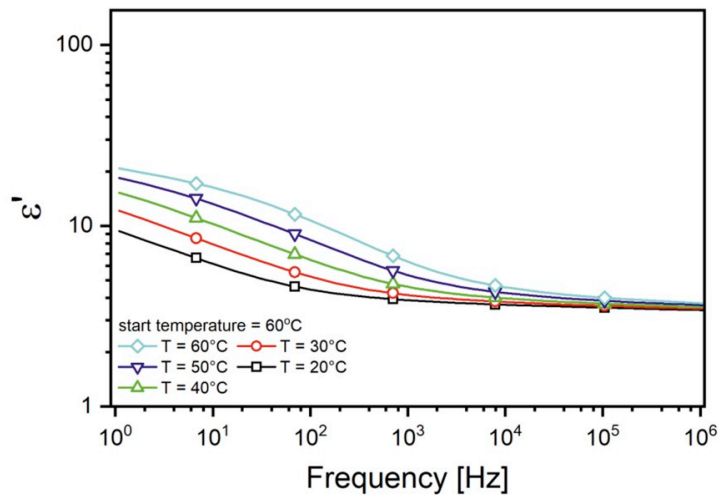

(b)

Fig. 4. Bode plots of $\varepsilon^{\prime}$ variation in the course of (a) 1st heating, (b) 1st cooling.

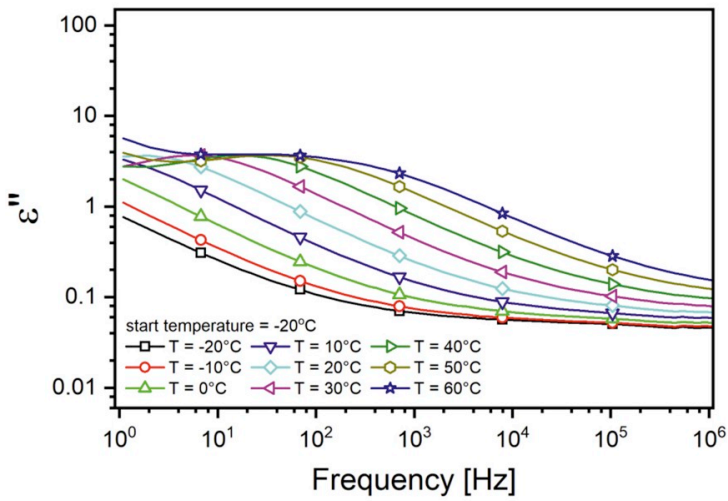

(a)

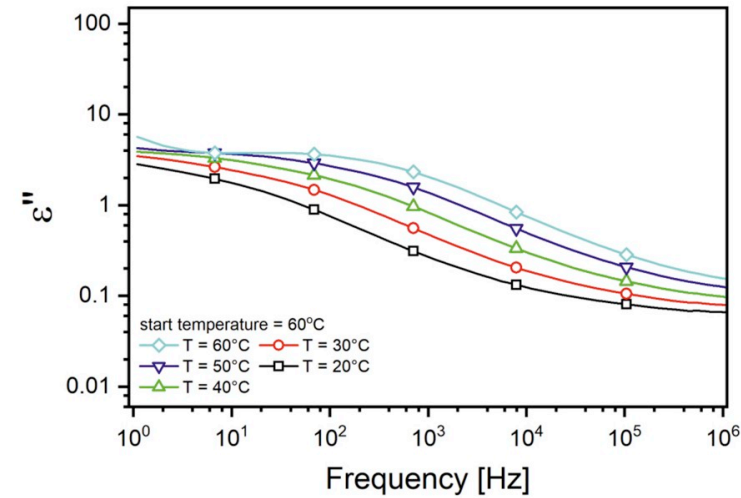

(b)

Fig. 5. Bode plots of $\varepsilon$ " variation in the course of (a) 1st heating, (b) 1st cooling.

accompanied with any change of $\varepsilon$ ' trace that reproduces the scheme observed at heating/cooling up to $80^{\circ} \mathrm{C}$.

The next heating/cooling cycle extended to $150^{\circ} \mathrm{C}$ has a completely new character (Fig. 11a). The shoulder appears in $\varepsilon$ " trace again at c.a. $110^{\circ} \mathrm{C}$. Next, at gradually raised temperatures, its maximum moves towards low frequencies, i.e. in the reverse direction that expected for a typical relaxation process. This time, unlike before, the process is accompanied with a distinct step-like change of $\varepsilon^{\prime}$ (Fig. 10a). As the temperature decreases, the shoulder (Fig. 11b) as well as the step threshold of $\varepsilon^{\prime}$ (Fig. 10b) move in the direction of lower frequencies, in a way characteristic of a normal relaxation process.

During the cooling this shoulder disappears at c.a $70^{\circ} \mathrm{C}$, then unexpectedly reappears $50^{\circ} \mathrm{C}$ (Figs. $11 \mathrm{~b}$ and $12 \mathrm{~b}$ ). The shoulder persisted at least for a couple of hours after completion of the cooling, when the sample was conditioned a regularly measured at $30^{\circ} \mathrm{C}$. The recorded $\varepsilon$, traces are also definitely different than that described previously 


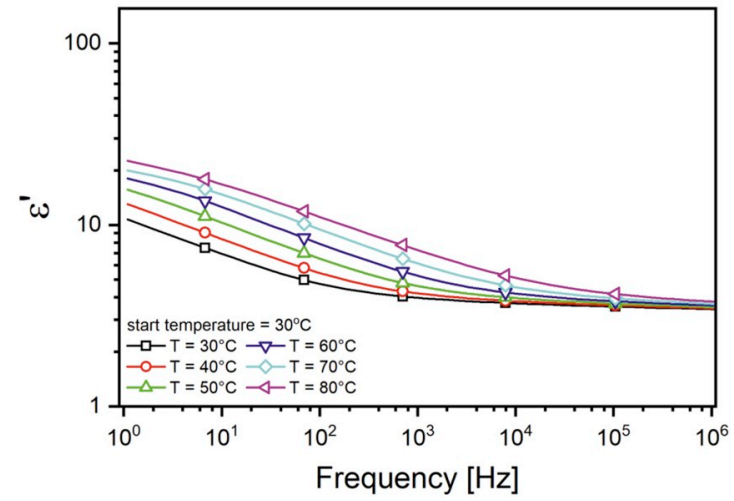

(a)

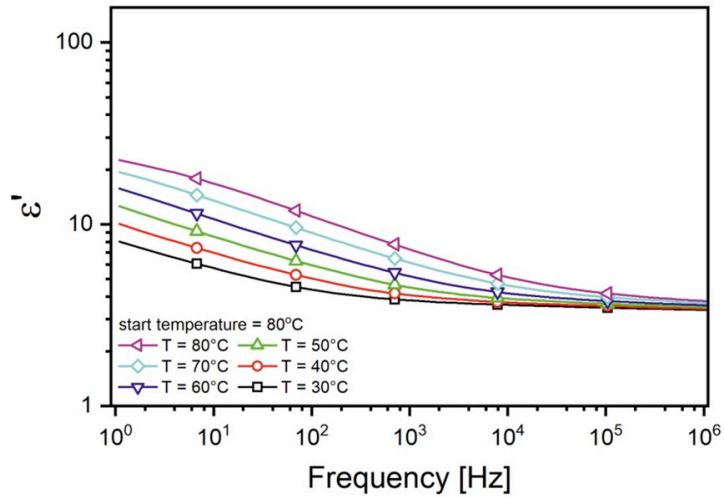

(b)

Fig. 6. Bode plots of $\varepsilon^{\prime}$ variation in the course of (a) 2nd heating, (b) 2nd cooling.

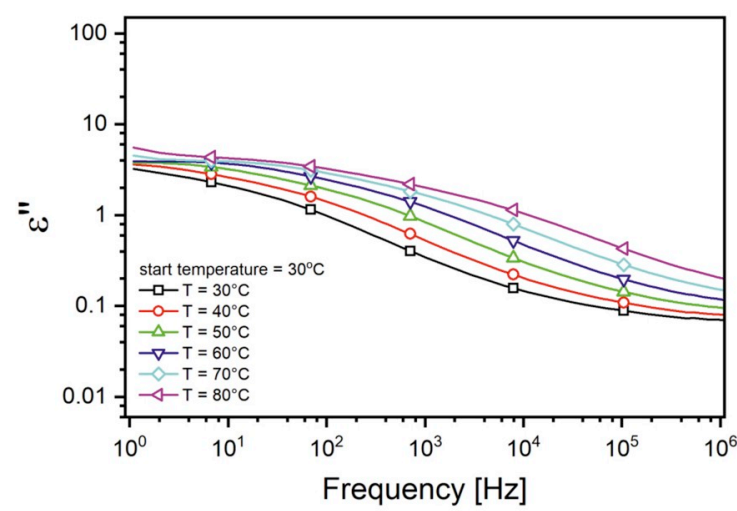

(a)

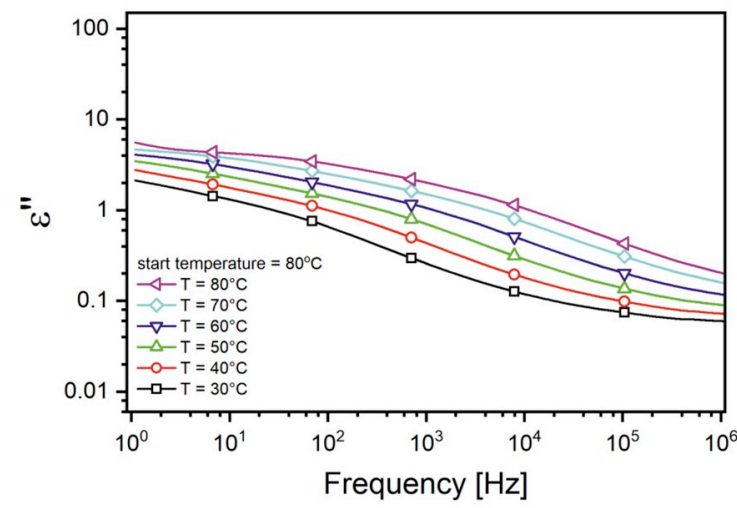

(b)

Fig. 7. Bode plots of $\varepsilon$ " variation in the course of (a) 2nd heating, (b) 2nd cooling.

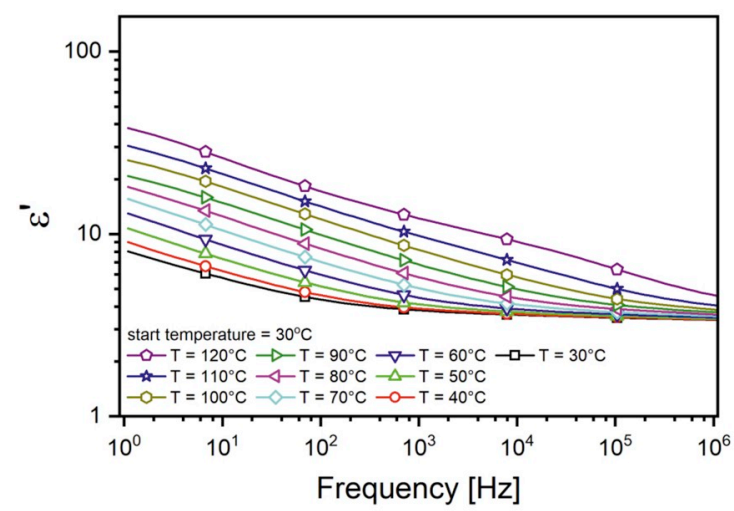

(a)

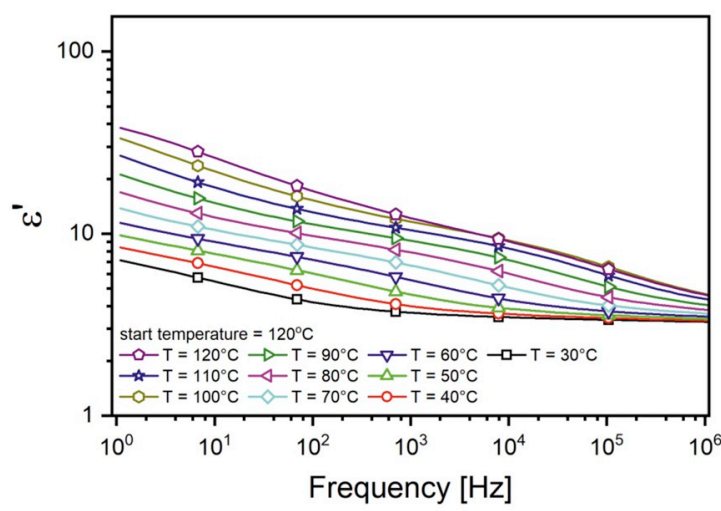

(b)

Fig. 8. Bode plots of $\varepsilon^{\prime}$ variation in the course of (a) 3rd heating, (b) 3rd cooling.

(Fig. 12a).

The data provided by dielectric spectroscopy may be presented in a more visually convincing manner in terms of the loss tangent (dissipation factor), defined as $\tan \delta=\varepsilon^{\prime \prime} / \varepsilon^{\prime}$. Its value doesn't depend on sample geometry what is an important asset [32]. Maps, illustrating the dependence of $\tan \delta$ on frequency and temperature are shown in Fig. 13 (for the 3rd heating/cooling cycle) and in Fig. 14 (for the 4th heating/cooling cycle). If one compares Fig. 13a and b, it can be concluded that the two images are very similar by the character, beside of a negligible difference in values of $\tan \delta$. This observation is very true, particularly for lower temperatures.

Fig. 14 contains contrasting images with those shown in Fig. 13. Already in the course of heating, an additional peak, visible as an island on the map, appears in vicinity of $10^{5} \mathrm{~Hz}$ (Fig. 14a). During the cooling the peak maintains intensity and shifts towards lower frequencies, as predicted by Arrhenius law. Below $60^{\circ} \mathrm{C}$ the peak separates in two parts. The first one continues to move toward lower frequencies, while the other and travels rightwards, ending at $10^{2} \mathrm{~Hz}$, when temperature reaches $30^{\circ} \mathrm{C}$. The picture emerging from the analysis in terms of tan $\delta$ is consistent with that obtained for permittivity and dielectric loss. 


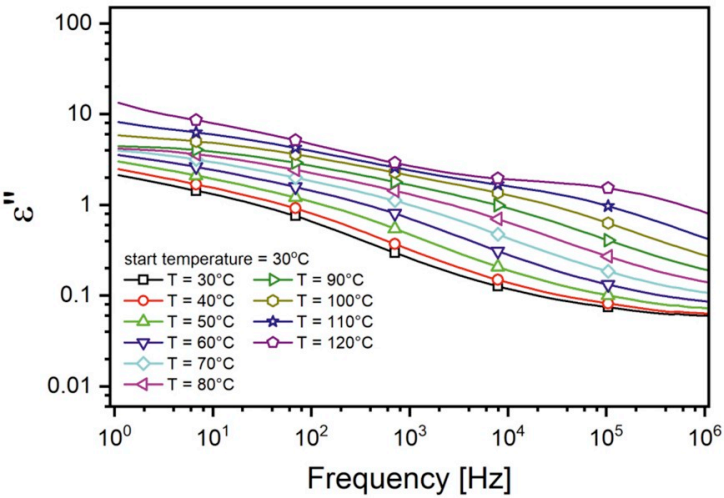

(a)

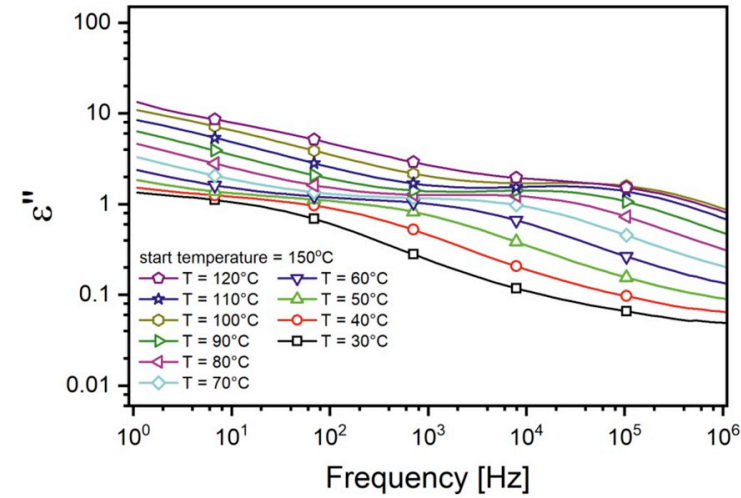

(b)

Fig. 9. Bode plots of $\varepsilon$ " variation in the course of (a) 3rd heating, (b) 3rd cooling.

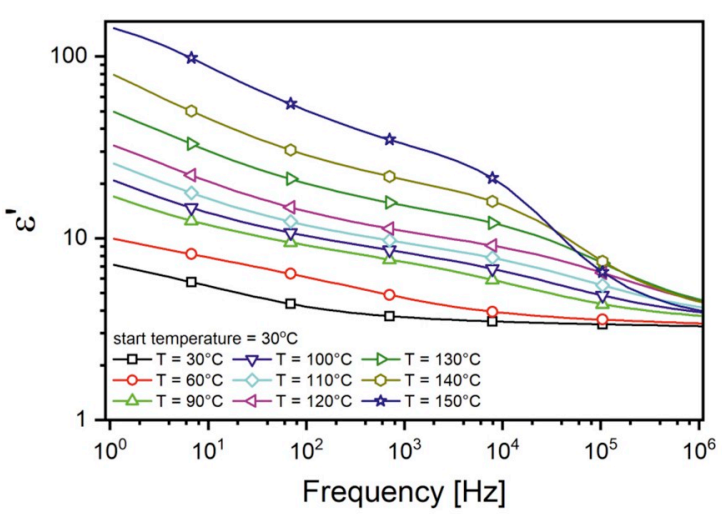

(a)

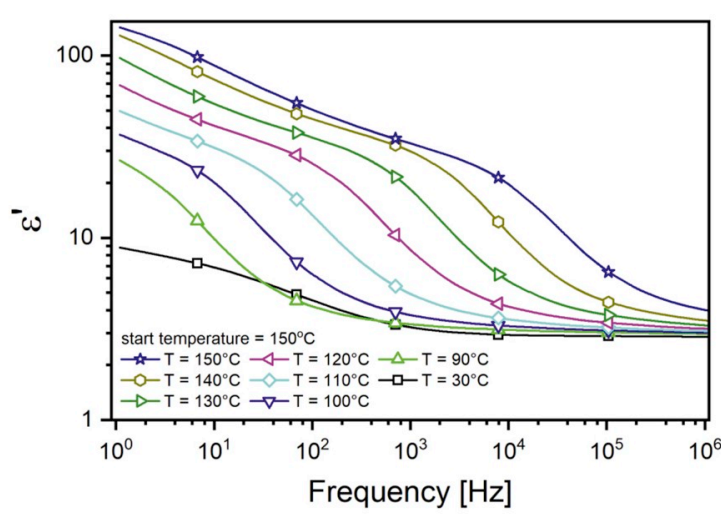

(b)

Fig. 10. Bode plots of $\varepsilon^{\prime}$ variation in the course of (a) 4th heating, (b) 4th cooling. For visual clarity, closely lying curves were not all shown.

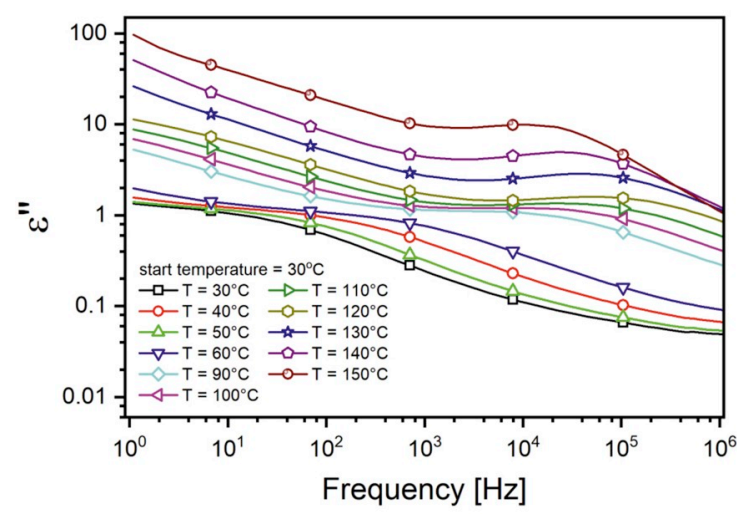

(a)

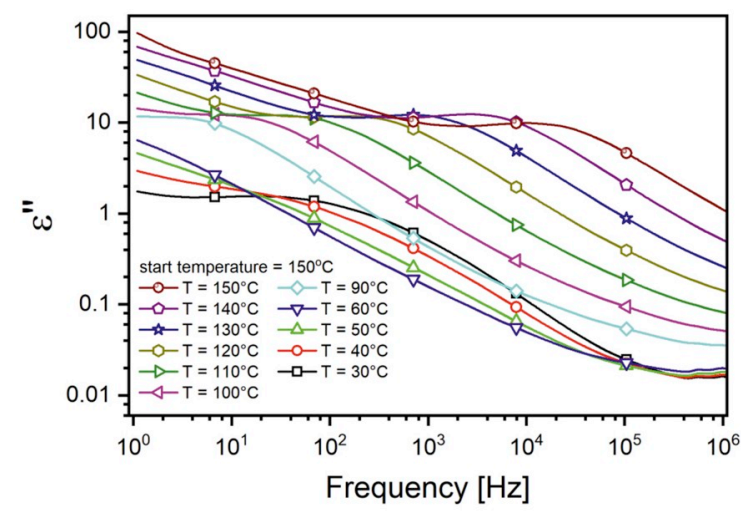

(b)

Fig. 11. Bode plots of $\varepsilon$ " variation in the course of (a) 4th heating, (b) 4th cooling. For visual clarity, closely lying curves were not all shown.

All the reported above observations confirm an important structural change occurring in DNA heated at increased temperatures between $120^{\circ} \mathrm{C}$ and $150^{\circ} \mathrm{C}$. This change can be associated with thermal denaturation of DNA double helix. Results obtained for the two other tablets were qualitatively identical, so they will not be discussed.

To confirm the hypothesis, put forward above, tablets, after completion of the heating/cooling routine, were dissolved in Tris buffer and next subjected to electrophoresis together with a reference material. The picture of the stained gel (Fig. 15), evidences the absence of double stranded DNA in thermally treated samples, at least of a length close to the initial (tens of base pairs).

The second assessment was performed with help of UV-vis absorption spectroscopy. The purity of DNA is routinely evaluated by the ratio of absorbance at $260 \mathrm{~nm}$ and $280 \mathrm{~nm}$. It is approximately equal to the weighted average of the A260/A280 ratios for the four nucleotides present. Pure DNA preparations have an A260/A280 ratio of greater than or equal to 1.8 , depending on actual composition of the nucleic acid. The ratio A260/A230 is used as a secondary measure of nucleic acid purity. Expected values are commonly in the range of 2.0-2.2. If the ratio is appreciably lower in either case, it may indicate the presence of 


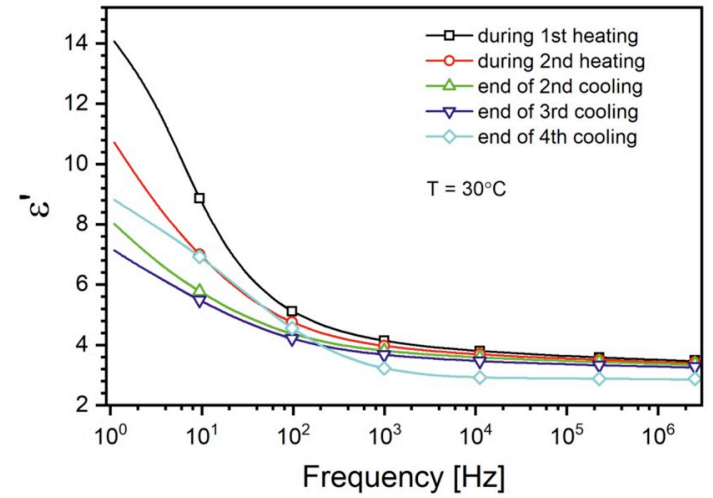

(a)

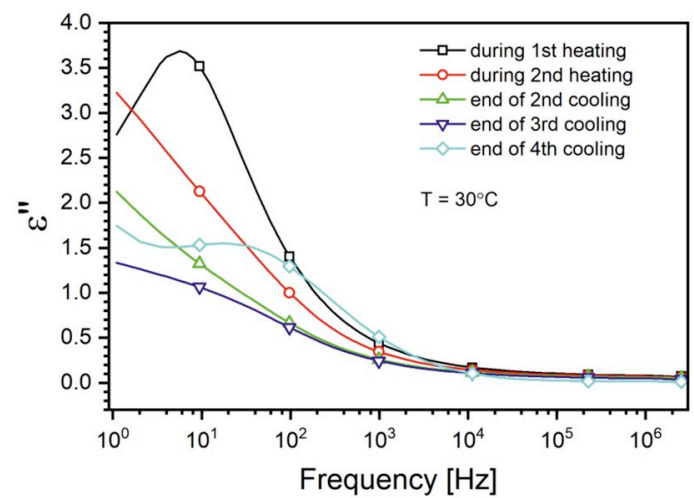

(b)

Fig. 12. Bode plots of (a) $\varepsilon^{\prime}$, (b) $\varepsilon^{\prime \prime}$ recorded at $\mathrm{T}=30^{\circ} \mathrm{C}$, shown in semi-logarithmic scale.

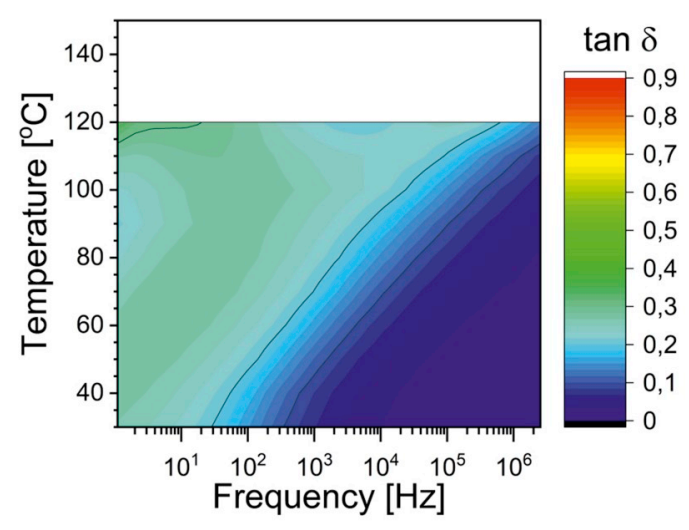

(a)

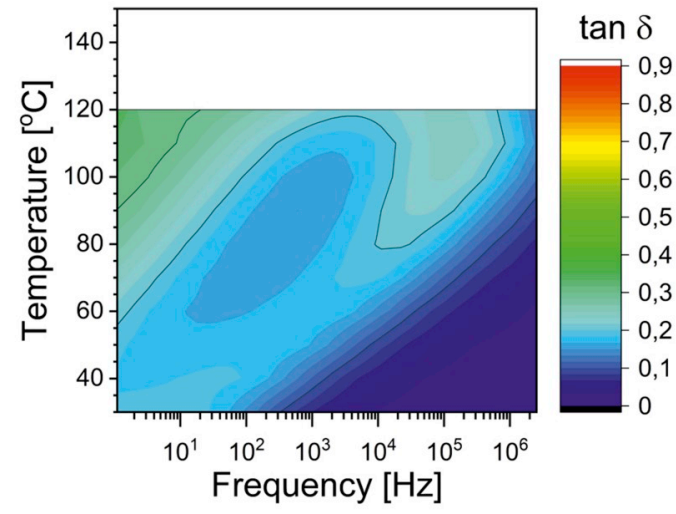

(b)

Fig. 13. Dependence of $\tan \delta$ on frequency and temperature. (a) 3rd heating cycle, from $30^{\circ} \mathrm{C}$ to $120^{\circ} \mathrm{C}$, (b) 3 rd cooling cycle, from $120^{\circ} \mathrm{C}$ to $30^{\circ} \mathrm{C}$.

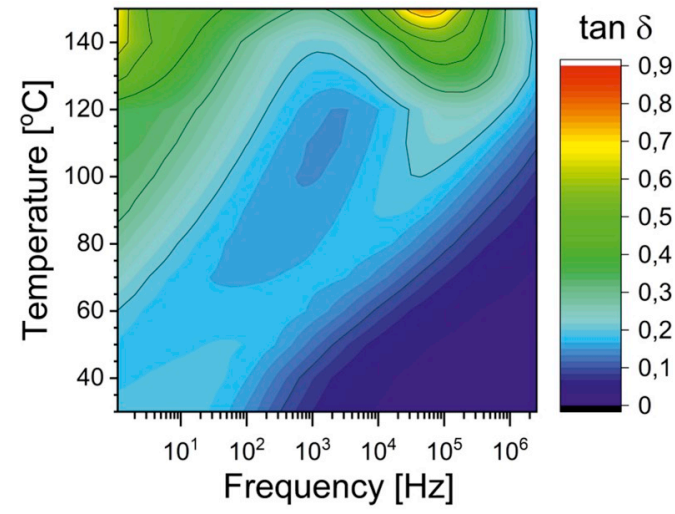

(a)

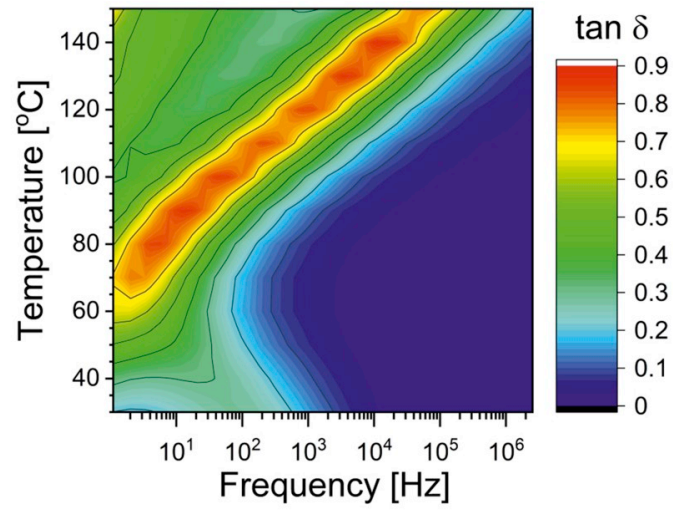

(b)

Fig. 14. Dependence of tan $\delta$ on frequency and temperature. (a) 4 th heating cycle, from $30^{\circ} \mathrm{C}$ to $150{ }^{\circ} \mathrm{C}$, (b) 4 th cooling cycle, from $150{ }^{\circ} \mathrm{C}$ to $30^{\circ} \mathrm{C}$.

contaminants that absorb strongly near $280 \mathrm{~nm}$ and $230 \mathrm{~nm}$, respectively [16].

Data presented in Table 1 support the conclusion drawn from electrophoretic research. At the end of the heating/cooling treatment, the DNA sample consisted mostly of single strands. Without additional tests, the kinetics of the observed phenomenon cannot be fully and unequivocally understood. However, a purely speculative model can be imaged as follows. Starting at $120^{\circ} \mathrm{C}$, as the temperature rises, DNA helices of increasing length separate. Thus, the average relaxation time increases, what explains the shift of $\alpha$-relaxation peak towards lower frequencies. During the cooling process, only single strands are present in the sample, so it resembles a linear polymer from morphological point of view. The question about the length of single strands remains open, as well about the origin of the onset peak appearing at the end of the 4th cooling (Fig. 11b). 


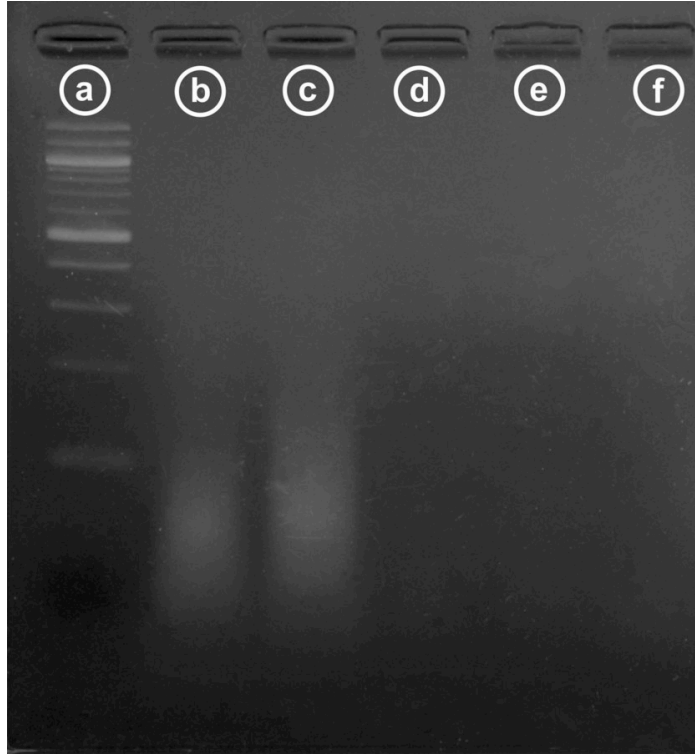

Fig. 15. Photography of UV illuminated, stained agarose gel containing specimens not treated thermally: (a) calibration ladder 100bp, (b) non-heated DNA tablet 50 Bar, (c) original DNA powder, next, DNA tablets after being heated up to $140{ }^{\circ} \mathrm{C}$ (d) 20 Bar, (e) 50 Bar, (f) 30 Bar.

Table 1

Results of UV-vis absorption tests.

\begin{tabular}{lll}
\hline Sample & A260/A280 & A260/A230 \\
\hline DNA powder, as delivered & 1.87 & 2.27 \\
DNA tablet, not heated & 1.9 & 2.19 \\
DNA tablet, heated at $150^{\circ} \mathrm{C}$ & 1.61 & 1.62 \\
\hline
\end{tabular}

\section{Conclusions}

Dielectric spectroscopy has proven to be an interesting alternative method for studying changes in DNA exposed to elevated temperatures. The reported research, established thermal stability limit at $120^{\circ} \mathrm{C}$ for the solid, biological double strand DNA. At higher temperatures the test samples got permanently denatured. No evidence of renaturation was found, at least for a few hours. This conclusion is in line with results obtained for double strand DNA thin films studied by spectroscopic ellipsometry [31]. However, kinetic factor seems to be of vital importance for DNA degradation. In the reported research samples were heated for "sufficiently long" time. In the case of a thermal stress applied for much shorter periods, DNA can resist higher temperatures as it was described in [23]. Nevertheless, the quantitative interpretation of the presented phenomenon requires much more extensive and detailed research.

\section{Declaration of competing interest}

On behalf of all the authors, I declare that there doesn't exist any kind of conflict of interest related to the submitted article.

\section{Appendix A. Supplementary data}

Supplementary data to this article can be found online at https://doi. org/10.1016/j.polymertesting.2019.106158.

\section{References}

[1] E.F. Gomez, A.J. Steckl, Engineering DNA and nucleobases for present and future device applications, in: M. Irimia-Vladu, E.D. Glowacki, N.S. Sariciftci, S. Bauer (Eds.), Green Materials for Electronics, Wiley-VCH, 2018, pp. 191-233.

[2] F. Zhang, J. Nangreave, Y. Liu, H. Yan, Structural DNA nanotechnology: state of the art and future perspective, J. Am. Chem. Soc. 136 (2014) 11198-11211.

[3] M. Falk, K.A. Hartman, R.C. Lord, Hydration of deoxyribonucleic acid. I. A gravimetric study, J. Am. Chem. Soc. 84 (1962) 3843-3846.

[4] H. Haranczyk, J. Czak, P. Nowak, J. Niziol, Initial phases of DNA rehydration by NMR and sorption isotherm, Acta Phys. Pol., A 117 (2010) 397-402.

[5] W. Saenger, W.N. Hunter, O. Kennard, DNA conformation is determined by economics in the hydration of phosphate groups, Nature 324 (1986) 385-388.

[6] I. Son, Y.L. Shek, D.N. Dubins, T.V. Chalikian, Hydration changes accompanying helix-to-coil DNA transitions, J. Am. Chem. Soc. 136 (2014) 4040-4047.

[7] R.S. Dias, K. Dawson, M. Miguel, Interaction of DNA with surfactants in solution, in: R.S. Dias, B. Lindman (Eds.), DNA Interactions with Polymers and Surfactants, Wiley, 2008, pp. 89-117.

[8] H. Haranczyk, J. Kobierski, D. Zalitacz, P. Nowak, A. Romanowicz, M. Marzec J. Niziol, E. Hebda, J. Pielichowski, Rehydration of CTMA modified DNA powders observed by NMR, Acta Phys. Pol., A 121 (2012) 485-490.

[9] J. Nizioł, H. Harańczyk, J. Kobierski, E. Hebda, J. Pielichowski, B. Ostachowicz, Hydration effect on solid DNA-didecyldimethylammonium chloride complexes measured using 1H-nuclear magnetic resonance spectroscopy, J. Appl. Phys. 114 (2013) 144701-144707.

[10] C. Leal, H. Wennerstrom, Hydration of DNA-amphiphile complexes, in: R.S. Dias, B. Lindman (Eds.), DNA Interactions with Polymers and Surfactants, Wiley, 2008, pp. 239-251.

[11] J. Nizioł, P. Nowak, J. Kobierski, H. Harańczyk, Temperature evolution of hydration shells in solid DNA didecyldimethylammonium chloride complex studied by 1H NMR spectroscopy, Eur. Polym. J. 66 (2015) 301-306.

[12] F. Sebastiani, A. Pietrini, M. Longo, L. Comez, C. Petrillo, F. Sacchetti, A. Paciaroni, Melting of DNA nonoriented fibers: a wide-angle X-ray diffraction study, J. Phys. Chem. B 118 (2014) 3785-3792.

[13] J.C. Genereux, J.K. Barton, Mechanisms for DNA charge transport, Chem. Rev. 110 (2010) 1642-1662.

[14] L. Sznitko, K. Parafiniuk, A. Miniewicz, I. Rau, F. Kajzar, J. Niziol, E. Hebda, J. Pielichowski, B. Sahraoui, J. Mysliwiec, Influence of surfactant on dynamics of photoinduced motions and light emission of a dye-doped deoxyribonucleic acid, Opt. Mater. 35 (2013) 2389-2393.

[15] D. Wanapun, V.J. Hall, N.J. Begue, J.G. Grote, G.J. Simpson, DNA-based polymers as chiral templates for second-order nonlinear optical materials, ChemPhysChem 10 (2009) 2674-2678.

[16] G.M. Blackburn, M.J. Gait, D. Loakes, D. Williams, Nucleic Acids in Chemistry and Biology, Royal Society of Chemistry, 2006, p. 586

[17] D. Gai, Y.P. Chang, X.S. Chen, Origin DNA melting and unwinding in DNA replication, Curr. Opin. Struct. Biol. 20 (2010) 756-762.

[18] T. Dauxois, N. Theodorakopoulos, M. Peyrard, Thermodynamic instabilities in one dimension: correlations, scaling and solitons, J. Stat. Phys. 107 (2002) 869-891.

[19] J.G. Duguid, V.A. Bloomfield, J.M. Benevides, G.J. Thomas Jr., DNA melting investigated by differential scanning calorimetry and Raman spectroscopy, Biophys. J. 71 (1996) 3350-3360.

[20] L. Wang, J. Yoshida, N. Ogata, Self-assembled supramolecular films derived from marine deoxyribonucleic acid (DNA) cationic surfactant complexes: large-scale preparation and optical and thermal properties, Chem. Mater. 13 (2001) 1273-1281.

[21] J.-I. Jin, J. Grote, Materials Science of DNA, 1 ed., CRC Press, 2011.

[22] J. Nizioł, J. Fiedor, J. Pagacz, E. Hebda, M. Marzec, E. Gondek, I.V. Kityk, DNAhexadecyltrimethyl ammonium chloride complex with enhanced thermostability as promising electronic and optoelectronic material, J. Mater. Sci. Mater. Electron. 28 (2017) 259-268.

[23] M. Karni, D. Zidon, P. Polak, Z. Zalevsky, O. Shefi, Thermal degradation of DNA, DNA Cell Biol. 32 (2013) 298-301.

[24] F. Delport, J. Pollet, K. Janssen, B. Verbruggen, K. Knez, D. Spasic, J. Lammertyn, Real-time monitoring of DNA hybridization and melting processes using a fiber optic sensor, Nanotechnology 23 (2012).

[25] I. Belozerova, D. Ge, R. Levicky, Electrochemical measurements of DNA melting on surfaces, in: P. Bergese, K. Hamad-Schifferli (Eds.), Nanomaterial Interfaces in Biology. Methods and Protocols, Humana Press, 2013, pp. 127-136.

[26] L. Yan, H. Iwasaki, Thermal denaturation of plasmid DNA observed by atomic force microscopy, Jpn. J. Appl. Phys., Part 1: Regular Papers and Short Notes and Review Papers 41 (2002) 7556-7559.

[27] A. Wildes, N. Theodorakopoulos, J. Valle-Orero, S. Cuesta-López, J.L. Garden, M. Peyrard, Thermal denaturation of DNA studied with neutron scattering, Phys. Rev. Lett. 106 (2011), 048101.

[28] J. Valle-Orero, J.L. Garden, J. Richard, A. Wildes, M. Peyrard, Glassy behavior of denatured DNA films studied by differential scanning calorimetry, J. Phys. Chem. B 116 (2012) 4394-4402

[29] J.D. Fredericks, P. Bennett, A. Williams, K.D. Rogers, FTIR spectroscopy: a new diagnostic tool to aid DNA analysis from heated bone, Forensic Sci. Int.: Genetics 6 (2012) 375-380.

[30] K. Imaizumi, Forensic investigation of burnt human remains, Res. Rep. Forensic Med. Sci. 5 (2015) 67-74.

[31] J. Nizioł, K. Makyła-Juzak, M.M. Marzec, R. Ekiert, M. Marzec, E. Gondek, Thermal stability of the solid DNA as a novel optical material, Opt. Mater. 66 (2017) 344-350. 
[32] F. Kremer, A. Schonhals, Broadband Dielectric Spectroscopy, Springer, 2002.

[33] G. Kortaberria, A. Jimeno, P. Arruti, A. Tercjak, M. Blanco, I. Mondragon, Dynamics of deoxyribonucleic acid as studied by dielectric spectroscopy, in: G. Kortaberria (Ed.), Biopolymers and Nanocomposites as Studied by Dielectric Spectroscopy Transworld Research Network, 2009.
[34] J.S. Mitchell, J. Glowacki, A.E. Grandchamp, R.S. Manning, J.H. Maddocks, Sequence-dependent persistence lengths of DNA, J. Chem. Theory Comput. 13 (2017) 1539-1555

[35] J.R. Brody, S.E. Kern, History and principles of conductive media for standard DNA electrophoresis, Anal. Biochem. 333 (2004) 1-13. 